\title{
Contribution of a New Mutation in parE to Quinolone Resistance in Extended-Spectrum- $\beta$-Lactamase-Producing Escherichia coli Isolates ${ }^{\nabla}$
}

\author{
Antonio Sorlozano, ${ }^{1}$ Jose Gutierrez, ${ }^{1 *}$ Antonio Jimenez, ${ }^{2}$ Juan de Dios Luna, ${ }^{3}$ and José Luis Martínez ${ }^{2}$ \\ Departments of Microbiology ${ }^{1}$ and Biostatistics, ${ }^{3}$ School of Medicine, University of Granada, and \\ Department of Urology, San Cecilio University Hospital, ${ }^{2}$ Granada, Spain
}

Received 31 May 2007/Accepted 5 June 2007

\begin{abstract}
Mutations in the quinolone resistance-determining regions of $\operatorname{gyr} A, \operatorname{gyr} B, \operatorname{par} C$, and $\operatorname{par} E$ were studied in 30 fluoroquinolone-resistant clinical isolates of Escherichia coli producing extended-spectrum $\beta$-lactamases. Ten isolates showed a mutation in parE that was significantly associated with an increase in the MIC for fluoroquinolones.
\end{abstract}

Spain has one of the highest rates of resistance to quinolones among European countries. Queipo-Zaragoza et al. (11) reported a significant increase in resistance to quinolones among clinical isolates of E. coli from 1990 (3\%) to 1998 (23\%). In 2003, resistance to ciprofloxacin in clinical isolates of $E$. coli obtained from blood cultures reached $19 \%$ in Spain, exceeded only by the reported rate in Portugal (9). However, these data are very likely biased (1).

There has also been an increase in the use of fluoroquinolones, especially cephalosporins, to treat enterobacterial infections, which has in turn increased the appearance of extendedspectrum $\beta$-lactamases (ESBLs) among these infections. The association between ESBL production and fluoroquinolone resistance is well documented (8) and may be due in part to the joint transfer of both mechanisms via plasmids (10). Nevertheless, this explains only a minimal proportion of the coresistance found.

The objective of this study was to investigate the presence of mutations in regions that code for quinolone resistance in the chromosomal genes gyrA, gyrB, parC, and parE in ESBL-producing clinical isolates of Escherichia coli.

Thirty previously characterized (13-15) ESBL-producing clinical isolates of $E$. coli were characterized for fluoroquinolone resistance using a microdilution assay for ciprofloxacin and levofloxacin (2). Reference strains Klebsiella pneumoniae ATCC 700603 and E. coli ATCC 25922 served as controls in all MIC determinations. PCR and direct DNA sequencing were used to identify mutations. The oligonucleotide primers $5^{\prime}$ ACGTACTAGGCAATGACTGG-3' and 5'-AGAAGTCGC CGTCGATAGAAC-3' were used to amplify nucleotides of gyr $A$, generating a fragment of $189 \mathrm{bp} ; 5^{\prime}$-TGTATGCGATGTC TGAACTG-3' and 5'-CTCAATAGCAGCTCGGAATA3' were used for parC (264-bp fragment); and 5'-TACCGAG CTGTTCCTTGTGG-3' and 5'-GGCAATGTGCAGACCAT CAG-3' were used for parE (266-bp fragment), following a previously described procedure (4). Finally, the oligonucleo-

\footnotetext{
* Corresponding author. Mailing address: Departamento de Microbiología, Facultad de Medicina, Avda de Madrid 11, E-18012 Granada, Spain. Phone: 349582435 50. Fax: 349582461 19. E-mail: josegf @ugr.es.

$\nabla$ Published ahead of print on 13 June 2007.
}

tide primers 5'-CTCCTCCCAGACCAAAGACA-3' and 5'TCACGACCGATACCACAGCC-3' were used for $g y r B$ (447-bp fragment), following a previously described procedure (17). Amplified DNA products were resolved by electrophoresis in agarose $(1.6 \%$, wt/vol) gels containing ethidium bromide. The PCR product was purified (Wizard PCR Preps DNA purification system; Promega, Madison, WI), processed with a DNA sequencing kit, and analyzed in an automatic DNA se-

TABLE 1. MIC (in $\mu \mathrm{g} / \mathrm{ml}$ ) for ciprofloxacin and levofloxacin and mutations detected in each gene of $E$. coli isolates

\begin{tabular}{|c|c|c|c|c|}
\hline \multirow{2}{*}{ Isolate } & \multicolumn{2}{|c|}{ MIC $(\mu \mathrm{g} / \mathrm{ml})$ of: } & \multicolumn{2}{|c|}{ Mutation in: ${ }^{a}$} \\
\hline & Ciprofloxacin & Levofloxacin & $\operatorname{parC}$ & parE \\
\hline 1 & 16 & 8 & S80I & - \\
\hline 25 & 8 & 4 & $\mathrm{E} 84 \mathrm{~K}$ & - \\
\hline 26 & 32 & 16 & S80I & - \\
\hline 41 & 32 & 8 & S80I & S458A \\
\hline 46 & 64 & 16 & S80I & S458A \\
\hline 58 & 4 & 4 & S80I & - \\
\hline 65 & 8 & 8 & S80I & - \\
\hline 101 & 8 & 8 & S80I & - \\
\hline 102 & 8 & 8 & S80I & - \\
\hline 115 & 8 & 4 & S80I & - \\
\hline 140 & 4 & 4 & S80R & - \\
\hline 141 & 8 & 4 & S80R & - \\
\hline 153 & 16 & 8 & S80I & - \\
\hline 159 & 64 & 16 & S80I & - \\
\hline 179 & 64 & 16 & S80I, E84V & - \\
\hline 180 & 32 & 16 & S80I, E84G & - \\
\hline 185 & 32 & 16 & S80I & - \\
\hline 189 & 128 & 16 & S80I & S458A \\
\hline 191 & 32 & 16 & S80I, A108V & - \\
\hline 195 & 64 & 16 & S80I & S458A \\
\hline 200 & 128 & 32 & S80I & S458A \\
\hline 207 & 64 & 16 & S80I & S458A \\
\hline 217 & 16 & 8 & S80I, A108V & - \\
\hline 219 & 16 & 8 & S80I & - \\
\hline 224 & 128 & 8 & S80I & S458A \\
\hline 226 & 32 & 8 & S80I & S458A \\
\hline 232 & 64 & 16 & S80I & S458A \\
\hline 237 & 32 & 32 & S80I & - \\
\hline 245 & 32 & 16 & S80I & S458A \\
\hline 255 & 8 & 8 & S80I & - \\
\hline
\end{tabular}

${ }^{a}$ _, absence of mutation. All isolates had mutations resulting in $\mathrm{S} 83 \mathrm{~L}$ and $\mathrm{D} 87 \mathrm{~N}$ in gyrA and no mutation in gyrB. 
TABLE 2. Spearman's coefficient (rho) values and significance for the relationship between MICs for ciprofloxacin or levofloxacin and the number of accumulated mutations for each isolate ${ }^{a}$

\begin{tabular}{|c|c|c|c|c|c|c|}
\hline \multirow[b]{2}{*}{ Drug } & \multicolumn{2}{|c|}{$\operatorname{par} C$} & \multicolumn{2}{|c|}{ parE } & \multicolumn{2}{|c|}{ All } \\
\hline & $\begin{array}{c}\text { Spearman's } \\
\text { coefficient } \\
\text { (rho) }\end{array}$ & $\begin{array}{l}\text { Significance } \\
\text { (bilateral) }\end{array}$ & $\begin{array}{c}\text { Spearman's } \\
\text { coefficient } \\
\text { (rho) }\end{array}$ & $\begin{array}{l}\text { Significance } \\
\text { (bilateral) }\end{array}$ & $\begin{array}{c}\text { Spearman's } \\
\text { coefficient } \\
\text { (rho) }\end{array}$ & $\begin{array}{c}\text { Significance } \\
\text { (bilateral) }\end{array}$ \\
\hline Ciprofloxacin & 0.104 & 0.584 & 0.689 & 0.000 & 0.722 & 0.000 \\
\hline Levofloxacin & 0.199 & 0.292 & 0.370 & 0.044 & 0.485 & 0.007 \\
\hline
\end{tabular}

${ }^{a}$ Determinations were not made for gyrA and $g y r B$ because the number of mutations was the same for all isolates (two in gyr $A$ and none in gyrB).

quencer (Applied Biosystems, Foster City, CA). E. coli strain 1-319, supplied by Jordi Vila of the University of Barcelona, was used as a control in the sequencing procedure (16). The sequence obtained for each of the genes in each of the 30 isolates was compared with the nucleotide sequence of the quinolone resistance-determining region (QRDR) of the reference strain $E$. coli K-12.

The Spearman correlation coefficient was used to explore relationships between the MICs of ciprofloxacin or levofloxacin and the numbers of mutations in each gene and in all four genes and to establish the direction of any relationship found.

Table 1 shows MICs for ciprofloxacin and levofloxacin obtained by microdilution and lists the mutations detected in each gene.

Table 2 displays the results of the statistical analysis, showing that the only correlation coefficients significantly different from 0 were those for the number of mutations in parE and the total number of mutations in relation to the MICs of both antibiotics.

The frequency of ESBL-producing strains detected in our region in 2002 (15) was much higher than that found in the same area by a Spanish multicenter study in 2000 (5). A similar finding was reported for quinolone resistance $(3,15)$. Besides the passage of time, the precise reasons for this increase are not clear.

Two mutations, encoding Ser83Leu and Asp87Asn, were detected in the QRDR of gyrA in all 30 E. coli isolates studied. The additional mutation in the Asp87 codon of gyrA is associated with a greater increase in fluoroquinolone resistance (17). However, since all isolates possessed these two mutations, it cannot be concluded that these changes are responsible for the differences in MICs for ciprofloxacin and levofloxacin among these isolates.

Six different mutations were observed in the QRDR of parC in these E. coli isolates: they encoded Ser80Ile (76.7\% of isolates), Ser80Arg (6.7\%), Glu84Lys (3.3\%), Ser80Ile and Glu84Gly (3.3\%), Ser80Ile and Glu84Val (3.3\%), and Ser80Ile and Ala108Val (6.7\%). Even though four isolates possessed two mutations and the rest showed a single substitution, from a statistical standpoint, it cannot be stated that these changes were in themselves responsible for the difference in MICs for fluoroquinolones among the different isolates.

Regarding parE, a similar study in Spain found no changes in the QRDR, although the authors did not rule out the possibility of other changes outside this region (12). In fact, 10 $(33.3 \%)$ of the present $E$. coli isolates showed a single mutation in parE, encoding Ser458Ala. A different mutation outside the QRDR, found in the same codon as that for Ser458Thr, was previously reported by Komp et al. (7). Statistical analysis of the relationship between the increase in MICs for ciprofloxacin and levofloxacin among the studied isolates and the presence of the Ser458Ala mutation showed significance, i.e., the increase in MICs was significantly related to the presence of this change. Seven mutations in parE have been described to date (6). The DNA sequence of parE is very similar to that of $g y r B$; therefore, parE mutations may also be associated with quinolone resistance. As all of these mutations have been detected outside the QRDR of parE, their contribution to quinolone resistance requires further investigation (6).

Other mechanisms implicated in fluoroquinolone resistance were not considered in the present study and may be responsible for the differences in MICs for fluoroquinolones observed among the isolates with the same types of mutation in all genes. Mutations in DNA gyrase, whether associated with mutations in topoisomerase IV or not, are an important determining factor for high levels of fluoroquinolone resistance. Changes in permeability, active expulsion of the antibiotic, and, to a lesser degree, the presence of plasmids are complementary mechanisms that cannot be excluded and may enhance development of resistance and contribute to the selection of fluoroquinolone-resistant isolates in the course of treatment with these antibiotics.

\section{REFERENCES}

1. Alos, J. I. 2003. Quinolones. Enferm. Infecc. Microbiol. Clin. 21:261-267.

2. Clinical and Laboratory Standards Institute. 2006. Methods for dilution antimicrobial susceptibility tests for bacteria that grow aerobically, 7th ed. Approved standard. CLSI publication M7-A7. Clinical and Laboratory Standards Institute, Wayne, Pa.

3. Daza, R., J. Gutierrez, and G. Piedrota. 2001. Antibiotic susceptibility of bacterial strains isolated from patients with community-acquired urinary tract infections. Int. J. Antimicrob. Agents 18:211-215.

4. Everett, M. J., Y. F. Jin, V. Ricci, and L. J. Piddock. 1996. Contributions of individual mechanisms to fluoroquinolone resistance in 36 Escherichia coli strains isolated from humans and animals. Antimicrob. Agents Chemother. 40:2380-2386.

5. Hernandez, J. R., A. Pascual, R. Canton, L. Martinez-Martinez, and Grupo de Estudio de Infeccion Hospitalaria. 2003. Extended-spectrum beta-lactamase-producing Escherichia coli and Klebsiella pneumoniae in Spanish hospitals (GEIH-BLEE Project 2002). Enferm. Infecc. Microbiol. Clin. 21:77-82

6. Hopkins, K. L., R. H. Davis, and E. J. Threlfall. 2005. Mechanisms of quinolone resistance in Escherichia coli and Salmonella: recent developments. Int. J. Antimicrob. Agents 25:358-373.

7. Komp, L. P., A. Karlsson, and D. Hughes. 2003. Mutation rate and evolution of fluoroquinolone resistance in Escherichia coli isolates from patients with urinary tract infections. Antimicrob. Agents Chemother. 47:3222-3232

8. Lautenbach, E., B. L. Strom, W. B. Bilker, J. B. Patel, P. H. Edelstein, and N. O. Fishman. 2001. Epidemiological investigation of fluoroquinolone resistance in infections due to extended-spectrum $\beta$-lactamase producing Escherichia coli and Klebsiella pneumoniae. Clin. Infect. Dis. 33:1288-1294.

9. Oteo, J., and J. Campos. 2004. Quinolone use and resistance. Enferm. Infecc. Microbiol. Clin. 22:201-203.

10. Poirel, L., J. D. Pitout, L. Calvo, J. M. Rodriguez-Martinez, D. Church, and 
P. Nordmann. 2006. In vivo selection of fluoroquinolone-resistant Escherichia coli isolates expressing plasmid-mediated quinolone resistance and expanded-spectrum $\beta$-lactamase. Antimicrob. Agents Chemother. 50:15251527.

11. Queipo-Zaragoza, J. A., A. Budía-Alba, M. J. Mascaros-Garcia, A. GomezFerrer, M. Gobernado, and J. F. Jimenez-Cruz. 2000. Evolución de la resistencia microbiana a fluorquinolonas en un hospital terciario. Actas Urol. Esp. 24:381-387.

12. Ruiz, J., S. Casellas, M. T. Jimenez de Anta, and J. Vila. 1997. The region of the parE gene, homologous to the quinolone-resistant determining region of the gyrB gene, is not linked with the acquisition of quinolone resistance in Escherichia coli clinical isolates. Antimicrob. Chemother. 39:839-840.

13. Sorlozano, A., J. Gutierrez, F. Fernandez, M. J. Soto, and G. Piedrola. 2004 A preliminary study on the presence of extended-spectrum beta-lactamases (ESBL) in clinical isolates of Escherichia coli in Granada (Spain). Ann. Microbiol. 54:227-232.

14. Sorlozano, A., J. Gutierrez, J. D. Luna, J. Oteo, J. Liebana, M. J. Soto, and
G. Piedrola. 23 March 2006, posting date. High presence of extended-spectrum $\beta$-lactamases and resistance to quinolones in clinical isolates of Esch erichia coli. Microbiol. Res. doi:10.1016/j.micres.2006.02.003. [Epub ahead of print.]

15. Sorlozano, A., J. Gutierrez, M. Palanca, M. J. Soto, and G. Piedrola. 2004 High incidence of extended-spectrum beta-lactamases among outpatien clinical isolates of Escherichia coli: a phenotypic assessment of NCCLS guidelines and a commercial method. Diagn. Microbiol. Infect. Dis. 50:131134.

16. Vila, J., J. Ruiz, P. Goñi, and M. T. de Anta. 1996. Detection of mutations in parC in quinolone-resistant clinical isolates of Escherichia coli. Antimicrob. Agents Chemother. 40:491-493.

17. Vila, J., J. Ruiz, F. Marco, A. Barcelo, P. Goñi, E. Giralt, and T. Jimenez de Anta. 1994. Association between double mutation in gyrA gene of ciprofloxacin resistant clinical isolates of Escherichia coli and MICs. Antimicrob. Agents Chemother. 38:2477-2479. 